\title{
Clinical analysis of the rap stress stimulator applied for crus fracture after skeletal external fixation
}

\author{
Ping Zhuang, Jiayuan Hong, Wei Chen, Jin Wu, Zhenqi Ding
}

Department of Orthopaedics, the $175^{\text {th }}$ Hospital of PLA, Orthopaedic Center of PLA, Southeast Hospital of Xiamen University, Zhangzhou, China

Submitted: 27 January 2013

Accepted: 23 June 2013

Arch Med Sci 2015; 11, 3: 612-618

DOI: 10.5114/aoms.2015.52366

Copyright $\odot 2015$ Termedia \& Banach

\section{Abstract}

Introduction: Open crus fracture is still difficult in clinical treatment because of the delayed fracture union and high rate of nonunion after the operation. A consensus has been reached that mechanical stress can promote fracture healing. We independently developed a stress stimulator, which can provide longitudinal pressure for the fixed fracture end of the lower legs to promote fracture healing. The purpose of this study is to explore the advantages and clinical effect of the rap stress stimulator applied for open crus fracture after skeletal external fixation.

Material and methods: One hundred and sixty-five patients (183 limbs) who suffered from open tibia and fibula fracture received skeletal external fixation, of which 108 limbs were treated with the rap stress stimulator after external fixation and 75 limbs were treated with regular functional exercises of muscle contraction and joint activity only. Then the fracture healing time and rate of nonunion were compared between the two groups.

Results: The mean fracture healing time and rate of nonunion in the group treated with the rap stress stimulator were $138.27 \pm 4.45$ days and $3.70 \%$ respectively, compared to $153.43 \pm 4.89$ days and $10.67 \%$ in the group treated without the stimulator.

Conclusions: The rap stress stimulator significantly shortened the fracture healing time and reduced the rate of nonunion for treating open tibia and fibula fractures.

Key words: stress stimulator, open tibia and fibula fracture, fracture healing.

\section{Introduction}

Open tibia and fibula fracture caused by high-energy injures is complex due to it being often accompanied by severe soft tissue impairment, and is vulnerable to infection and avascular necrosis of soft tissue. It is well accepted that the skeletal external fixator used for open tibia and fibula fracture is a simple and effective method to enable safe healing. However, it is still difficult in clinical treatment because of the delayed fracture union and high rate of nonunion after the operation [1, 2]. Its rates of delayed fracture healing and nonunion after the operation were reported to be $18 \%$ [3] and 5\% [4] respectively. Once nonunion has occurred, a bone graft and internal fixation are required. However, due to the significant scars, it is difficult to carry out skin suture in the secondary operation. Moreover, postoperative complications such as skin flap

\author{
Corresponding author: \\ Zhenqi Ding \\ Department of Orthopaedics \\ the $175^{\text {th }}$ Hospital of PLA \\ Orthopaedic Center of PLA \\ Southeast Hospital \\ of Xiamen University \\ No.369, Zhanghua Road \\ 363000 Zhangzhou (Fujian), \\ China \\ Phone: +8613960084384 \\ E-mail:30851798@qq.com
}


edge necrosis or infection usually occur. Prolonged course of the disease induces complications such as ankylosis and muscular atrophy, which affect the restoration of limb function. After undergoing external fixation for open tibia and fibula fracture, patients were treated with the rap stress stimulator in our hospital. This report evaluated the clinical effect of the aforementioned treatment by comparative study.

\section{Material and methods}

\section{Instrument}

The self-developed rap stress stimulator (patent number: CN803117.5) is composed of a shell, horizontal-trench bracket, hammer, and control plane for driving the electric motor. The hammer rapped the heel to produce an intermittent rap force, causing the micromovement of the fracture end and generating the pressure. The control plane regulates the rap frequency and strength with a range of $0.1-10 \mathrm{~Hz}$ and 20 Newtons $(\mathrm{N})-$ $350 \mathrm{~N}$ respectively (Figure 1 ).

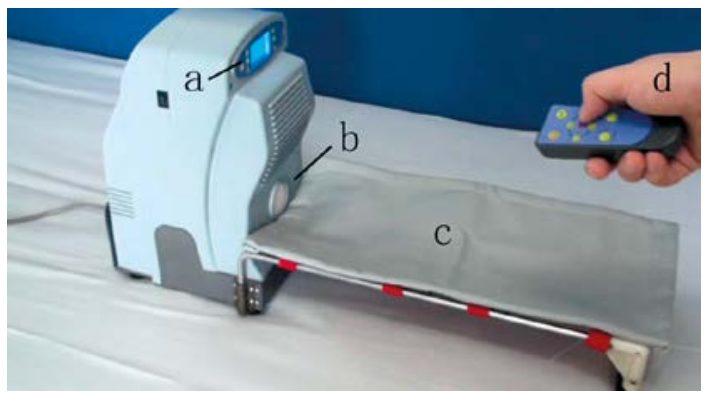

Figure 1 . The rap stress stimulator is shown

$a$-Control panel, $b$-hammer, $c$ - bracket, $d$-remote control, $e$-power switch, $f$-controlling button of strength of hammer acting force, $g$-controlling button of frequency of hammer acting force.
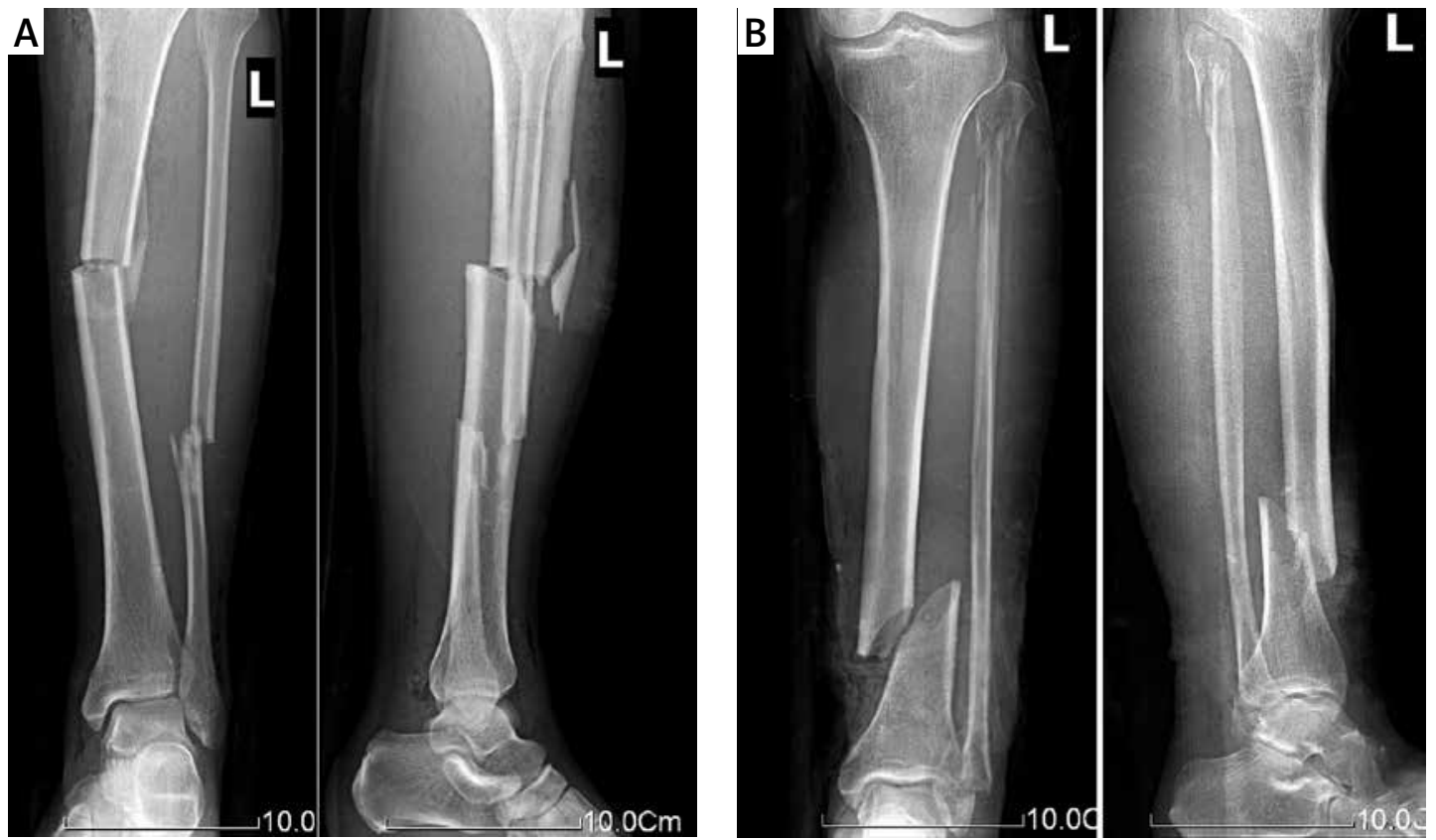

Figure 2. X-ray film of typical cases with open tibia and fibula fracture. A - Experimental group, B - control group 
Table I. Comparison of Hannover Fracture Scale, patients' age and duration of operation between two groups

\begin{tabular}{|lccc|}
\hline Group & HFS & Age [years old] & Duration of operation [min] \\
\hline Experimental & $16.4 \pm 3.5$ & $30.6 \pm 13.2$ & $75.2 \pm 17.8$ \\
\hline Control & $18.0 \pm 4.2$ & $33.4 \pm 15.2$ & $73.4 \pm 16.8$ \\
\hline & $p=0.12$ & $p=0.99$ & $p=0.86$ \\
\hline
\end{tabular}

We defined $p<0.05$ to be statistically significant.

39 multiple fractures of limbs, 8 pelvic fractures, 17 spinal fractures, 23 craniocerebral traumas, $11 \mathrm{ab}$ domen or thorax traumas. The surgical procedures were performed by the same group of surgeons. Patients were divided into two groups after the operation with 108 limbs treated with the rap stress stimulator in the experimental group, and 75 limbs in the control group were subjected to regular exercises of muscle contraction and joint activity. There were no statistically significant differences between the two groups in the Hannover Fracture Scale, patients' age or duration of operation $(p>0.05)$ (Table I).

\section{Operation method}

Patients consulted our hospital within 0.5-6 h after getting hurt, and their vital signs such as consciousness, blood pressure, pulse and respiration were under close observation. They had received emergency treatments including intravenous antibiotics, dressing wounds with sterile gauzes, fixation with temporary splints and symptomatic treatment such as anti-shock, hemostasis, etc. Surgery
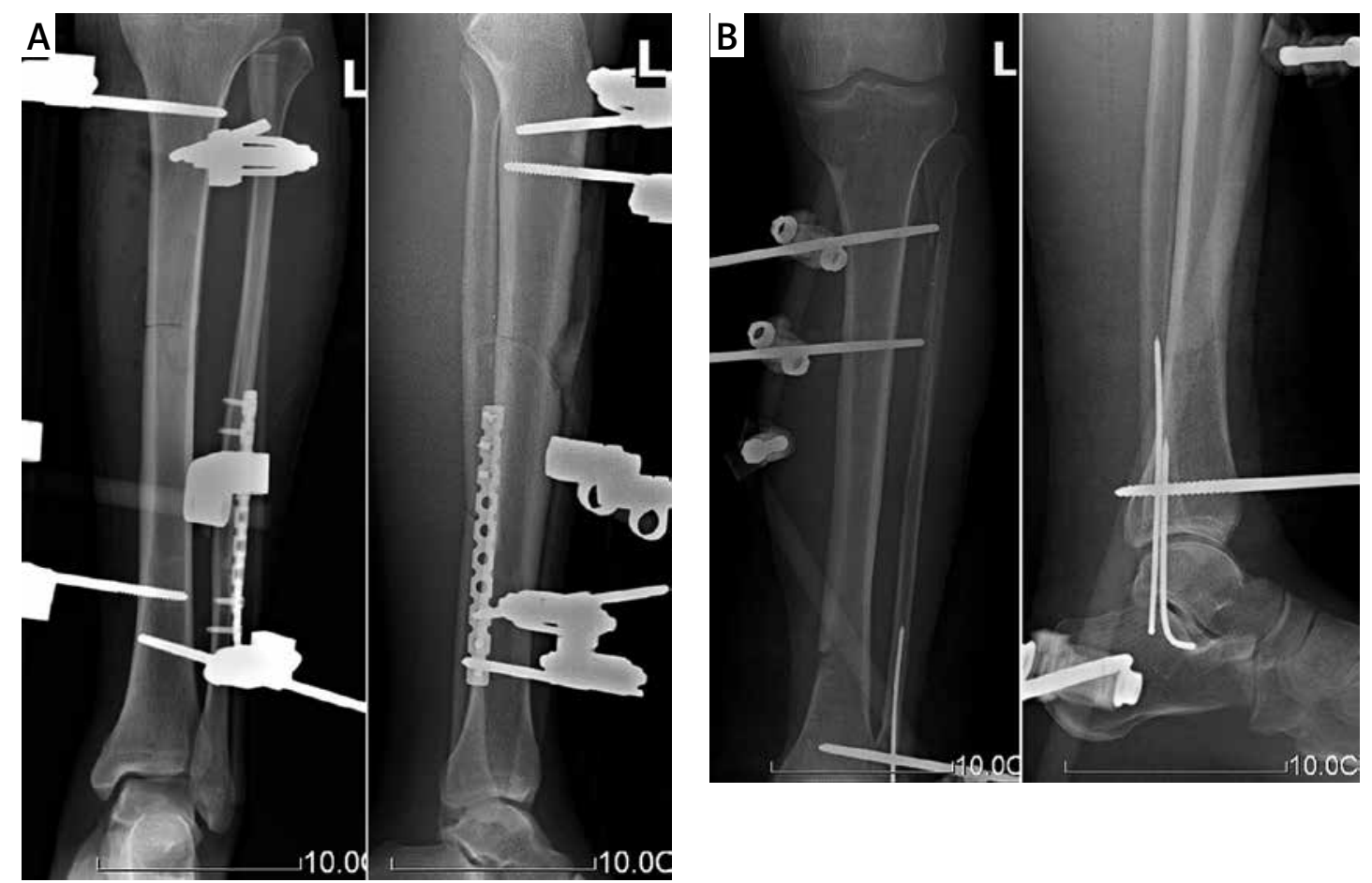

was performed with stable vital signs. During the surgery, foreign matter and nonviable tissue were removed thoroughly, vascular beds were restored, and soft tissue and periosteum with blood supply were protected. Then we adjusted alignment of the wounded limb in traction status and reduced the fracture ends, of which the unstable ones were treated with limited internal fixation through Kirchner wire or screws, which then were covered by soft tissue. In all the cases a unilateral skeletal external fixator was applied by screwing 2 or 3 fixation pins into the distal and proximal fracture of the tibia respectively. The fixation pin should avoid the wound as far as possible, and its length should be just sufficient to penetrate the opposite cortical bone. When fracture of the distal tibia occurred close to the ankle joint, it was treated with cross ankle joint fixation (Figure 3). After that, the external frame was fitted. If the fractures were accompanied by injuries of the tendon, nerves or vessels, restoration of these should be followed. Wounds were closed primarily as far as possible and drainage was applied. Relaxation suture or flap/muscle

Figure 3. Open tibia and fibula fracture treated with skeletal external fixation. The X-ray film was made immediately after the operation. A - Experimental group, B - control group 
Table II. Lane-Sandhu score

\begin{tabular}{|lr|}
\hline Score & Callus formation and fracture line \\
\hline 0 & No callus formation and fracture line was clear \\
\hline 1 & $25 \%$ callus formation and fracture line was relatively clear \\
\hline 2 & $50 \%$ callus formation and fracture line was obscure \\
\hline 3 & $75 \%$ callus formation and fracture line had basically vanished \\
\hline 4 & $100 \%$ callus formation and fracture line had completely vanished \\
\hline
\end{tabular}

flap transplant was selected according to the situation that the wounded limb swelling or local soft tissue defect was serious to close the wound.

\section{Postoperative treatment and assessment criteria}

Postoperative antibiotics and dehydrating agents were used and wounded limbs were lifted up. The swelling and peripheral blood circulation of the wounded limb were observed carefully. All patients were guided to exert muscle contraction and joint activity generally. Tenderness in the fracture area and longitudinal percussion pain were observed routinely by the visual analog scale (VAS) score of these patients at 3, 6 and 9 months postoperatively. We defined the VAS score from 0 to 10 points, indicating the pain level; a score of 0 indicated painless, while a score of 10 indicated acute pain that could not be endured any more. X-rays were rechecked regularly to examine the status of fracture healing, and we used the Lane-Sandhu score (Table II) to evaluate the callus formation rate and visible level of fracture line at the same time points as above. One week after the operation, 108 limbs in the experimental group were treated with the rap stress stimulator $(175 \mathrm{~N}, 0.5 \mathrm{~Hz}) 30$ min per time, 3 times per day. After these patients were discharged with a primary healed wound, they were guided to use the rap stress stimulator continually until the fracture clinical healing was completed (Figure 4).

\section{Statistic analysis}

All statistical analyses were performed using SPSS 13.0 software. The measurement data were compared using the $t$-test and enumeration data were compared using the chi-squared $\left(\chi^{2}\right)$ test. Statistical difference was accepted at $p<0.05$.

\section{Results}

All patients were followed up with an average of 13.3 months (range from 9 months to 3 years). $X$-ray imaging showing clearness of the fracture line, hardening of the fracture end, or sealing of the medullary cavity 9 months after the operation was performed to identify bone nonunion. In the experimental group (108 limbs), 4 limbs which were treated with bone graft and internal fixation had fracture nonunion, and no osteoporosis was found on the follow-up X-ray. In the control group (75 limbs), fracture nonunion was found in 8 limbs and the aforementioned method was carried out for them too. Furthermore, different degrees of osteoporosis were found. There was a significant difference between the experimental group and control group in the rate of nonunion, which was $3.70 \%$ and $10.67 \%$ respectively $(p<0.05)$. In the experimental group (108 limbs), 4 limbs had nonunion and the other 104 limbs reached primary clinical fracture union with a mean healing time of $138.27 \pm 11.45$ days, while in the control group (75 limbs), 8 limbs had nonunion and the other 67 limbs had a mean clinical fracture healing time of $153.43 \pm 12.89$ days. The results revealed that there were significant difference between the two groups in mean clinical fracture healing time $(p<0.05)$. At 3, 6 and 9 months postoperatively, VAS score and Lane-Sandhu score also showed a significant difference between the experimental group and the control group (Table III, Figure 5).

\section{Discussion}

How to improve the healing of open tibia and fibula fracture

Serious open tibia and fibula fracture always have distinct fracture displacement and severe soft tissue injury, and there was a high rate of

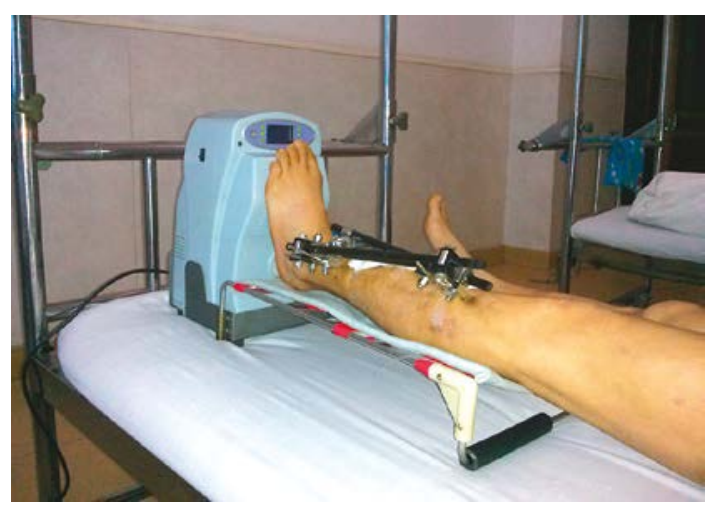

Figure 4. Treatment image of the rap stress stimulator is shown 
Table III. Results compared between experimental group and control group

\begin{tabular}{|lccc|}
\hline Variable & Experimental group & Control group & Value of $p$ \\
\hline Nonunion rate & $3.70 \%$ & $10.67 \%$ & $<0.0001$ \\
\hline Clinical fracture healing time (days + variance) & $138.27 \pm 11.45$ & $153.43 \pm 12.89$ & $<0.0001$ \\
\hline \begin{tabular}{l} 
VAS score (mean + variance): \\
\hline 3 months
\end{tabular} & $3.14 \pm 0.53$ & $4.05 \pm 0.62$ & $<0.0001$ \\
\hline 6 months & $1.25 \pm 0.75$ & $2.23 \pm 0.86$ & $<0.0001$ \\
\hline 9 months & $0.54 \pm 0.61$ & $0.92 \pm 0.57$ & $<0.0001$ \\
\hline Lane-Sandhu score (mean + variance): & $1.28 \pm 0.53$ & $1.06 \pm 0.52$ & 0.006 \\
\hline 3 months & $2.34 \pm 0.73$ & $1.95 \pm 0.82$ & 0.0009 \\
\hline 6 months & $3.50 \pm 0.88$ & $2.71 \pm 1.06$ & $<0.0001$ \\
\hline 9 months & & & \\
\hline
\end{tabular}

We defined $p<0.05$ to be statistically significant.
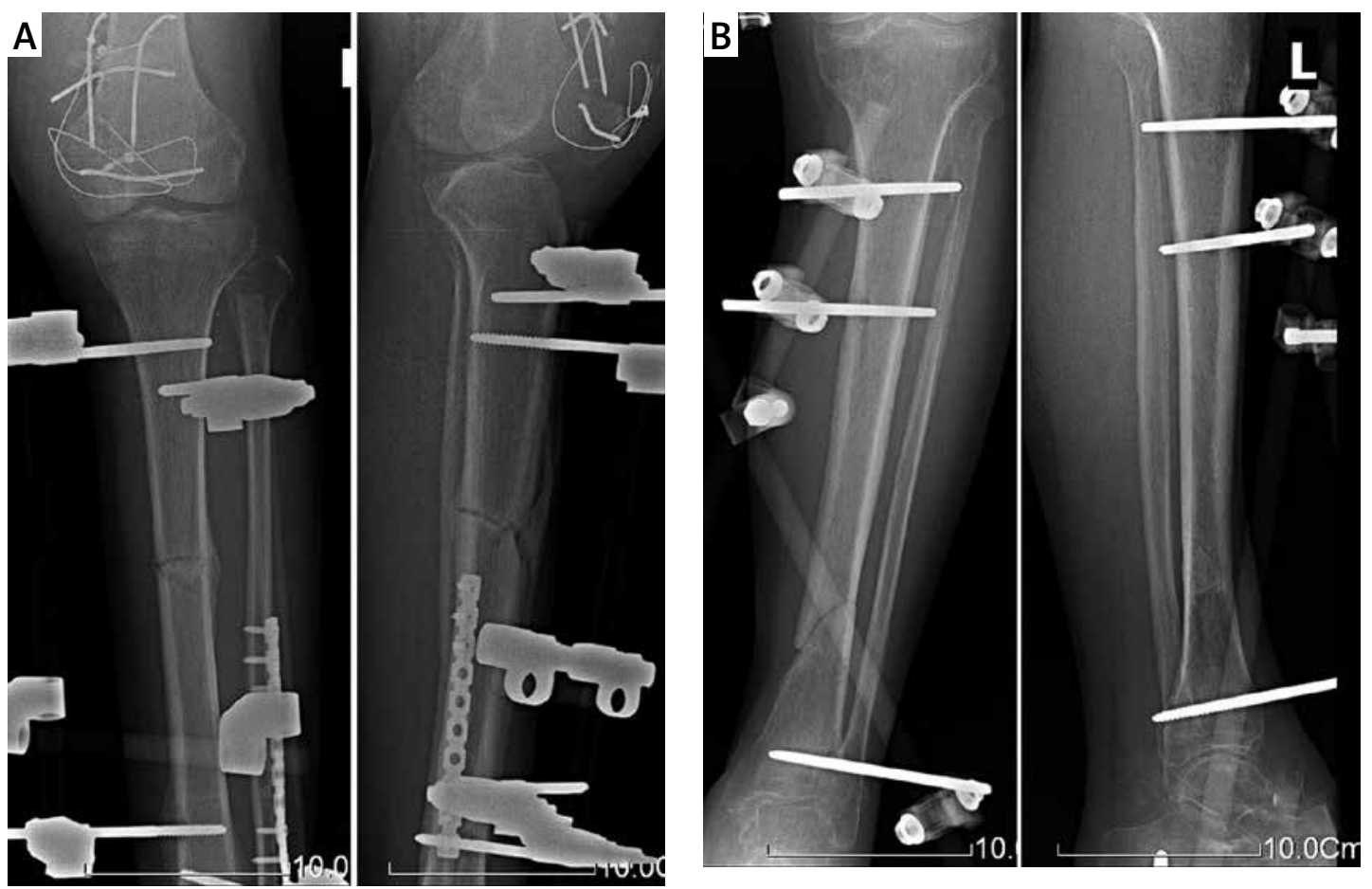

Figure 5. X-ray film of typical cases 3 months after the operation is shown. Obvious osteotylus is seen in experimental group (A) compared with control group (B)

delayed fracture healing or nonunion postoperatively. How to improve open tibia and fibula fracture healing stands out among the most important issues in orthopedics. Blood supply and fixation are basic conditions for fracture healing, and the stress environment around the fracture ends is the key to shorten the duration of fracture healing [5]. In the clinic, our hospital treats serious open tibia and fibula fracture with skeletal external fixation, which has almost no added disturbance of local blood circulation around the fracture ends, and this immobility fixation facilitates bone revascularization and creates favor- able conditions for fracture healing. Postoperative application of the rap stress stimulator can effectively solve the problems of stress deficiency caused by long periods of bed rest and stress shielding by fixation. Thus the fracture healing can be improved and its duration shortened with a satisfactory clinical result. In a word, application of skeletal external fixation combined with the rap stress stimulator can provide fixation and stress stimulation to fracture ends without further jeopardizing the local blood circulation, which creates favorable mechanical and biological conditions for fracture healing. 


\section{Analysis of the curative effect of the rap stress stimulator}

There is a consensus that stress stimulation can improve fracture healing [6-9]. The rap stress stimulator raps the heels through a hammer to generate a stress. The stress is conducted through the longitudinal axis of the lower limb and then generates pressure on the lower limb fracture end similarly as in walking. Thus, the biological and biomechanical environments in the fracture site are improved, which consequently facilitates fracture healing $[10,11]$. In the present study, the mean primary fracture healing time of the experimental group was $138.27 \pm 4.45$ days, and 6 limbs had nonunion (3.70\%), while in the control group, the mean primary healing time was $153.43 \pm 4.89$ days, and 11 limbs had nonunion (10.67\%). The results revealed significant differences between the two groups in mean fracture healing time and rate of nonunion $(p<0.05)$. It suggested that the rap stress stimulator can significantly shorten the duration of fracture healing and decrease the rate of nonunion, thus facilitating the recovery of injured limbs.

\section{Acceptance and exclusion criteria and comparability analysis of the experimental and control group}

The main objective of the present study was to explore the effect of the rap stress stimulator on fracture healing. Therefore, patients with other factors affecting fracture healing such as bone defect and postoperative infection were ruled out. Patients who underwent a secondary operation because of postoperative nonunion were also excluded. The mean primary fracture healing time of the two groups was compared. Because the healing time was unaffected by bone defect, postoperative infection and secondary operation, it can reflect the effect of the rap stress stimulator exactly.

\section{Strength, application time and orientation of stress improving fracture healing}

Only if stress on the fracture end and the rigidness of the healing tissues are balanced and coordinated can tissues differentiate well and heal. When this balance is achieved, the optimum stress strength is determined [12]. After fixation, the stress borne by fracture ends should be determined by axial stress loading vs. fixed rigidness. The skeletal external fixator applies non-rigid fixation and most energy generated from the rap heel can arrive at the fracture end. Therefore, the rap force should be controlled carefully in clinical practice to avoid exceeding tissue bearing capability, damaging the callus, and inducing a delayed union. In the present study, application of a rap with $175 \mathrm{~N}$ significantly shortened the duration of fracture healing, and thus clinical efficacy was favorable. However, whether $175 \mathrm{~N}$ is the best stress strength remains unclear, and further study is necessary.

The best application time of stress to facilitate fracture healing is in the early stage of healing. As we know, bio-reaction including cell activation and differentiation happens at this stage [13]. Morgan et al. [14] suggested that if physical stimulation were applied to treat the fracture clinically, the application time should be in the early stage of fracture healing, i.e. within 5 weeks after fracture, which will achieve marked effects. Chao et al. [15] also suggested that the most effective application time of stress was in the early stage of fracture healing before some special cells are activated or differentiation occurs. Therefore, in the present study, the rap stress stimulator was applied 1 week after the operation.

Axial stress drives osteoblast and fibroblast differentiation into the osteogenic aspect, so it is beneficial for fracture healing. However, shear force beyond a limit will jeopardize the fresh capillary and callus, and stimulate fibroblast proliferation to form fibro-tissue which affects fracture healing [15]. When using mechanical stress in clinical practice to promote fracture healing, a shear force on the fracture end often appeared when axial stimulation stress was applied to the fracture's end, and thus fracture redisplacement readily occurs, interfering with the fracture healing. For this reason, in the present study, the stress stimulator was supplemented following fracture fixation by the external fixator, so the stress was imposed to a steadily fixed fracture end. Then, the relatively small shearing force made by postoperative axial percussion can be eliminated by the fixator. The rigid fixation effectively overcomes the shearing force on the fracture's end, and the stress stimulator produces axial intermittent compressive stress by rapping the heel. Thus, the fracture's end obtains axial stress stimulation in a relatively stable status, which contributes to the healing of the fracture.

\section{Advantages of the rap stress stimulator}

Clinically, patients suffering from serious open crus fractures often also suffered from craniocerebral trauma, abdominal-thoracic trauma, pelvic fracture, spinal fracture, etc. They needed long periods of bed rest and could not perform early functional exercises, so the facture ends were in need of stress stimulation. The rap stress stimulator can be used early to facilitate fracture healing in such patients, and partly prevent delayed union or nonunion caused by deficient stress on the fracture end because of long periods of bed 
rest. The instrument is safe, noninvasive and simply controlled. Moreover, the stress level as well as the frequency can be intuitively adjusted. In clinical practice, the patient is able to set the mechanical parameters by him or herself. Consequently, our instrument is readily accepted by patients.

Long periods of fixation and lack of stress stimulation for fracture often lead to osteoporosis. However, in the present study, no apparent osteoporosis was found on the follow-up X-ray of patients treated with the postoperative rap stress stimulator. This suggested that the rap stress stimulator partly prevents disuse osteoporosis caused by long periods of fixation for fracture and lack of stress stimulation.

Application of the rap stress stimulator followed by external fixation to treat serious open crus fractures can speed up the fracture healing process. In the present study, the rap stress stimulator provided a force $(175 \mathrm{~N}, 0.5 \mathrm{~Hz})$ to rap the heel, which significantly shortened the fracture healing time without making patients uncomfortable. However, the optimum strength and frequency of stress were still undecided. In clinical treatment, the rap stress stimulator should be applied after a comprehensive analysis and overall judgments on the fracture classifications, features of impaired soft tissue, stability of surgical fixation, clinical examination and $X$-ray, etc. The rap strength should be adjusted step by step without making patients uncomfortable. With further improvement, the rap stress stimulator can be gradually applied in clinical practice to promote the healing of fractures and relieve the pain of patients.

\section{Conflict of interest}

The authors declare no conflict of interest.

\section{References}

1. Gold SM, Wasserman R. Preliminary results of tibial bone transports with pulsed low intensity ultrasound (Exogen). J Orthop Trauma 2005; 19: 10-6.

2. Deng Z, Cai L, Jin W, et al. One-stage reconstruction with open bone grafting and vacuum-assisted closure for infected tibial non-union. Arch Med Sci 2014; 10: 764-72.

3. Bhandari M, Guyatt GH, Swiontkowski MF, Schemitsch EH. Treatment of open fractures of the shaft of the tibia. J Bone Joint Surg Br 2001; 83: 62-8.

4. Einhorn TA. The cell and molecular biology of fracture healing. Clin Orthop Relat Res 1998; 355: S7-21.

5. Goodship AE, TJ Lawes, CT Rubin. Low-magnitude highfrequency mechanical signals accelerate and augment endochondral bone repair: preliminary evidence of efficacy. J Orthop Res 2009; 27: 922-30.

6. Baas E, Kuiper JH, Yang Y, Wood MA, El Haj AJ. In vitro bone growth responds to local mechanical strain in three-dimensional polymer scaffolds. J Biomech 2010; 43: 733-9.
7. Gonzalez-Torres LA, Gomez-Benito MJ, Doblare M, Garcia-Aznar JM. Influence of the frequency of the external mechanical stimulus on bone healing: a computational study. Med Eng Phys 2010; 32: 363-71.

8. Pal B, Gupta S, New AM, Browne M. Strain and micromotion in intact and resurfaced composite femurs: experimental and numerical investigations. J Biomech 2010; 43: 1923-30.

9. Yang X, Gong P, Ling Y, et al. Cyclic tensile stretch modulates osteogenic differentiation of adipose-derived stem cells via the BMP-2 pathway. Arch Med Sci 2010; 6: 152-9.

10. Tang N, Zhao Z, Zhang L, et al. Up-regulated osteogenic transcription factors during early response of human periodontal ligament stem cells to cyclic tensile strain. Arch Med Sci 2012; 8: 422-30.

11. Duan C, Liu J, Yuan Z, et al. Adenovirus-mediated transfer of VEGF in to marrow stromal cells combined with PLGA/TCP scaffold increases vascularization and promotes bone repair in vivo. Arch Med Sci 2014; 10: 147-81.

12. Foux A, Yeadon AJ, Uhtoff HK. Improved fracture healing with less rigid plates. Clin Orthop 1997; 339: 232-45.

13. Klein P, Schell H, Streitparth F, et al. The initial phase of fracture healing is specifically sensitive to mechanical conditions. J Orthop Res 2003, 21: 662-9.

14. Morgan EF, Salisbury Palomares KT, Gleason RE, et al. Correlations between local strains and tissue phenotypes in an experimental model of skeletal healing. J Biomech 2010; 43: 2418-24.

15. Chao EY, Aro HT, Lewallen DG, et al. The effect of rigidity on fracture healing in external fixation. Clin Orthop 1989; 241: 24-35. 